\title{
Biological Mechanisms of Lithium separately and in combination with Caffeine in Palatogenesis in Albino Rat
}

\author{
Hagar Abdel Sabour Saad ${ }^{(1)}$, Mona H M Farid $^{(2)}$, Heba Ahmed Adawy ${ }^{(3)}$
}

Codex : 55/1810

azhardentj@azhar.edu.eg

http://adjg.journals.ekb.eg

\section{KEYWORDS}

Lithium carbonate, Caffeine,

cleft palate, $\mathrm{Ki}-67$

\begin{abstract}
Purpose: The purpose of this study was to evaluate the Biological Mechanisms of Lithium separately and in combination with Caffeine in Palatogenesis in Albino Rat Materials and Methods: Thirty six adult rats were used in this study, twenty four female and twelve male rats, after mating procedures and confirmation of pregnancy the pregnant rats were divided into 3 groups :group1(control), which consists of six pregnant rats and were not receive any medication during gestation, group2' Which consists of nine pregnant rats and were received prianil (lithium carbonate) $200 \mathrm{mg} /$ $\mathrm{kg}$ intragasteric During the period of ( 6th to 15th ) day of gestation \&group 3: Which consists of nine pregnant rats and were received prianil (lithium carbonate) $200 \mathrm{mg} /$ $\mathrm{kg}$ rat and caffeine $18 \mathrm{mg} / \mathrm{kg}$ rat intragasteric durig the same period. The fetuses were obtained from mother were decapitated and the heads were fixed in buffered formaline for at least 3 hours. The specimens were collected, prepared and examined by routine haematoxylin \&eosin and immunostained by (Ki-67) marker. Results: at 16, 18 and 21 days of gestation, the greatest mean value was recorded in group II, followed by group III, with the least value recorded in group I. Both group I and III revealed a decrease in area percent of (Ki-67) throughout the study, whereas in group II revealed an increase. Conclusions: The study offers a clear evidence that lithium during pregnancy causing cleft palate in fetus and caffeine could efficiently antagonize the teratogenicity of high doses of lithium on palate when both administrated.
\end{abstract}

\section{INTRODUCTION}

In pregnancy, drug treatment presents a special concern due to the threat of potential teratogenic effects of the drug ${ }^{(1)}$. The physiology

- Paper extracted from master thesis entitled "Biological Mechanisms of Lithium separately and in combination with Caffeine in Palatogenesis in Albino Rat).

1. Demonstrator of Oral and Dental Biology, Oral and Dental Biology Department, Faculty of Dental Medicine, Al-Azhar University for Girls.

2. Professor and Head of Oral and Dental Biology Department, Faculty of Dental Medicine, Al- Azhar University for Girls.

3. Assistant Professor of Oral and Dental Biology, Oral and Dental Biology Department, Faculty of Dental Medicine, Al-Azhar University for Girls. 
of pregnancy affects the pharmacokinetics of medications used and certain medications can reach the fetus and cause harm ${ }^{(2)}$. All antipsychotics cross the placenta and, as such, consideration needs to be given to their potential to cause structural or functional dysgenesis of fetal organs and/or skeletal structures when exposure occurs in first trimester. It is generally considered that the baseline population rate for malformation in the general population is $1-3 \%{ }^{(3)}$. The administration of lithium results in several congenital defects in the fetus, including cleft palate ${ }^{(4)}$. It activates $\mathrm{Wnt} / \mathrm{b}$-catenin signaling by inhibiting GSK-3b ${ }^{(5)}$.

Cleft palate is a common congenital defect, affecting roughly one in 2000 newborn babies worldwide. The etiology of cleft includes multiple environmental and genetic factors ${ }^{(6,7)}$. Lithium compounds, also known as lithium salts, are primarily used as a psychiatric medication. This includes in the treatment of major depressive disorder that does not improve following the use of other antidepressants, and bipolar disorder (ASHSP, $2015)^{(8)}$. In these disorders, it reduces the risk of suicide ${ }^{(9)}$.

There are also drugs that can increase the clearance of lithium from the body, which can result in decreased lithium levels in the blood. These drugs include theophylline, caffeine, and acetazolamide. Additionally, increasing dietary sodium intake may also reduce lithium levels by prompting the kidneys to excrete more lithium ${ }^{(10)}$.

A recommendation to limit caffeine intake during pregnancy was issued by the United States Food and Drug Administration in 1980. More recently, the American Congress of Obstetricians and Gynecologists reported that moderate caffeine consumption ( $<200 \mathrm{mg} /$ day) during pregnancy does not seem to be a major risk factor of miscarriage or preterm birth ${ }^{(11)}$.

The purpose of the present study was to evaluate the Biological Mechanisms of Lithium separately and in combination with Caffeine in Palatogenesis in Albino Rat.

\section{MATERIALS AND METHODS:}

Thirty six adult rats were used in this study, twenty four female and twelve male rats, after mating procedures and confirmation of pregnancy the pregnant rats were divided into 3 groups:group1(control), which consists of six pregnant rats and were not receive any medication during gestation, group 2' Which consists of nine pregnant rats and were received prianil (lithium carbonate) $200 \mathrm{mg} / \mathrm{kg}$ intragasteric During the period of (6th to 15th ) day of gestation \& group 3: Which consists of nine pregnant rats and were received prianil (lithium carbonate) $200 \mathrm{mg} / \mathrm{kg}$ rat and caffeine $18 \mathrm{mg} / \mathrm{kg}$ rat intragasteric durig the same period. The fetuses were obtained from mother were decapitated and the heads were fixed in buffered formaline for at least 3 hours. The collected specimens were prepared for histological and immunohistochemical studies by( ki-67)marker.

\section{Evaluation of ki-67immunostaining by image analysis:}

The number of positively reacting immunostaining of ki-67 was counted. Counting was based on the area with the highest percentage of positive cells, each stained nucleus was regarded as positive regardless of the staining intensity. The sites expressing a positive immune reaction with the ki67 antibody are identified as brown deposits of the chromogen. The stronger the immune reaction, the darker the chromogen intensity.

\section{RESULTS}

In (group 1) at day 18 of gestation, The palatal shelves are completely fused and separated the oral and nasal cavities with completely disappearance of MES.The covering epithelium thins out and doesn't differentiate into stratified squamous epithelium toward the oral cavity in the midline, but in the 
lateral region the epithelium begins to differentiate While in (group 2), At this day it was found that 5 fetuses of total 22 displayed cleft palate while the remaining 18 fetuses showed fused palatine shelves. In (group 3), complete fusion of palatal shelves with each other and with the nasal septum and bone trabecule formation appeared beneath nasal cavity. The epithelium covering the palate toward the OC was still thin in the midline but becomes thick at the sides (Fig. 1).

In (group 1) at day 21 of gestation, The palatal shelves are completely fused and separated the oral and nasal cavities with completely disappearance of MES.The covering epithelium differentiate into stratified squamous epithelium toward the oral cavity in the midline, and in the lateral region. While in (group 2), At this day it was found fusion of palate with the persistence of the medial epithelial seam (MES) at line of fusion, MES consists of two layer of flattened epithelial cells which represents the edge epithelium of each palatine shelves. In (group 3), complete fusion of palatal shelves with each other and with the nasal septum and bone trabecule formation appeared beneath nasal cavity. The epithelium covering the palate toward the OC differentiates into keratinized stratified squamous epithelium becomes thick (Fig. 1).

Both (control) and (caffeine and lithium) groups revealed a decrease in area percent of Ki-67 throughout the study, whereas the (lithium) treated group revealed an increase. Comparing the percent change in area percent of Ki-67 throughout the study in different groups, One way analysis of variance (ANOVA) test revealed that the difference of mean value was statistically significant. Tukey's post hoc test revealed no significant difference between control and caffeine groups.(Fig.1(A-F))

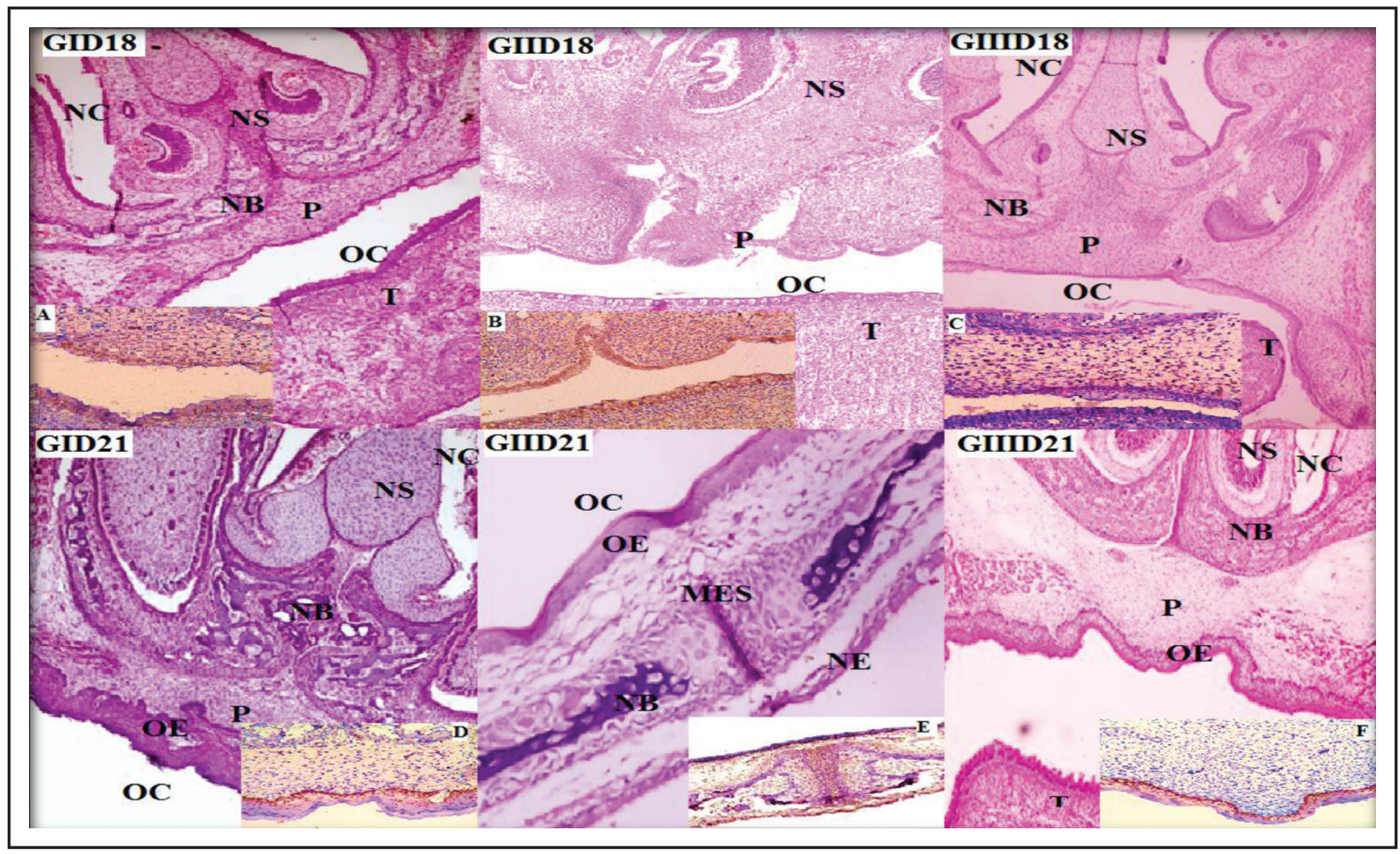

Fig. (1): GID18 (control at day 18), GIID18 (lithium group at day 18), GIIID 18 (lithium and caffeine group at day 18), GID21(control at day 21), GIID21 (lithium group at day 21), GIIID21 (lithium and caffeine group at day 21), NS(Nasal Septum), NB (Nasal Bone), P(Palate), T(Tongue), OC(Oral Cavity), NC(Nasal Cavity), OE(Oral Epithelium), NE(Nasal Epithelium). 


\section{DISCUSSION}

The choice of rats in this study was according to another study (12) who reported that the early development of the human face is essentially similar to that of the rat. Also physiological body functions of rats and human are nearly similar. Besides that, it has a short period of gestation period (18- 21 days).

In this study, the average dose of choice drug (prianil C-R ) for rats amounted to $200 \mathrm{mg} / \mathrm{kg}$ of weight per day. This dose was calculated according to the used equation by converting adult human therapeutic dose to animal dose $\mathrm{e}^{(13)}$. This dose resulting in cleft palate in $22.7 \%$ of fetuses accompanied by maternal mortality, miscarriage and premature labor. Similarly studies ${ }^{(\mathbf{1 4}, \mathbf{1 5})}$ agreed with our results .

Moreover, another study ${ }^{(16)}$ reported that lithium inhibits palatal fusion and osteogenic differentiation in palatal shelves in vitro.The chosen periods of intragastric administration of $200 \mathrm{mg} / \mathrm{kg}$ prianil (lithium carbonate) to pregnant rats were from 6 th to 15 th day of gestation as this was the organogenesis period of pregnancy in wistar rats ${ }^{(\mathbf{1 7})}$.

To trace the development of palate (palatogenesis) the fetuses of the control group were sacrificed at different successive periods begin at day 14 of gestation where the development begin. Then rats were sacrificed their fetuses were tacken at days 15 , 16,17 and18 of gestation to determine the position and fusion of the palatine shelves and at day 21 of gestation to determine whether the palatal formation completed or not yet. All these previous chosen periods was in accordance to others ${ }^{(18)}$.

In the present study the pregnant rats which received intragastric $200 \mathrm{mg} / \mathrm{kg}$ body weight of lithium at 6 to 15 days of pregnancy resulting in $22.7 \%$ of fetuses with cleft palate. These results revealed that, lithium at the present dose, period and route of administration obviously, interfere with the normal organogenesis and growth of the palatal shelves, retarded the fusion of PS through inhibition of glycogen synthase kinase 3 beta (GSK3b), resulting in persistence of MES. GSK3b plays an important role in the regulation of cell proliferation and apoptosis and the cell apoptosis is required for MES disappearance during palatal fusion. These result in agreement with others who reported that lithium inhibits palatal fusion and osteogenic differentiation in palatal shelves in vitro ${ }^{(16)}$.

On the other hands, the results of various studies (including cohort, prospective, retrospective and small number case reports) indicate that lithium is a "weak" teratogen in humans and animal studies with lithium using doses comparable to human therapeutic serum levels have not reported any abnormalities $^{(19)}$.

To interpret the failure of PS union in this study observed at 18th and 21th days this may be due to that lithium mediated GSK3b inhibition prevents palatal fusion and osteogenic differentiation by b-catenin signaling. Disturbance of the network of signaling pathways in palatogenesis may result in a failure of fusion of the palatal shelves, and hence in a cleft palate ${ }^{(20)}$.

In the present study, Lithium mediated GSK3b inhibition induced persistence of the MES, which suggests it can cause cleft. The result of this study is in agreement with previous studies in vivo and in vitro in mice ${ }^{(\mathbf{1 5 ) ( 1 6 )}}$.Closure and fusion of the secondary palate requires timed interactions, movements, and apoptosis along the medial margins of the palatal shelves ${ }^{(21)}$.

In group III which received lithium in combination with caffeine the palatal shelves were normally fused, with normal epithelial lining and without epithelial remnants in middle region. These findings revealed the enhancing effect of caffeine against lithium teratogenicity by decreasing lithium blood level through acceleration of its secretion. These results confirm the findings stated by many authors. ${ }^{(22,23)}$. On the other hand another study ${ }^{(24)}$ reported that even in low doses ( $4 \mathrm{mg} /$ day), caffeine suppresses the growth of embryonic and fetal tissues. 
By utilizing antibody Ki-67 which is a nuclear protein that is associated with cellular proliferation and it is also associated with ribosomal RNA transcription (25).Inactivation of antigen Ki-67 leads to inhibition of ribosomal RNA synthesis (26). Immunohistochemeical observation indicated that lithium carbonate increased the positive staining of ki-67 in both epithelium and connective tissues. A study (27) reported that there was an increase in the number of Ki-67-positive cells after administration of lithium for 14 days. another study (28) reported that chronic but not subacute treatment with antidepressants increase the cellular proliferation.

\section{CONCLUSIONS:}

From the present study, the following conclusions could be stated:

1. The lithium during pregnancy causing cleft palate in fetus, so during pregnancy, the benefits of medication need to be carefully weighed against risks for the mother, for the fetus, and of neonatal complications, as well as risks during breast-feeding. Accordingly, significant efforts have been made to define these risks for all medications available to pregnant women, including psychopharmacological medications

2. Caffeine could efficiently antagonize the teratogenicity of high doses of lithium on palate when both administrated in the early period of organogenesis.

\section{REFERENCES}

1. Bánhidy, F., Lowry, R. B., \& Czeizel, A. E. Risk and benefit of drug use during pregnancy. International Journal of medical sciences 2005; 2 : 100-6.

2. Deborah E, McCarter, Spaulding MS. Medications in pregnancy and lactation. Amer J Maternal Child Nursing 2005; 30: 10-7.

3. Galbally, M., Snellen, M., \& Power, J. Antipsychotic drugs in pregnancy: a review of their maternal and fetal effects. Therapeutic advances in drug safety 2014; 5: 100-109.
4. Sharma, A., \& Rawat, A. K. Teratogenic effects of lithium and ethanol in the developing fetus. Alcohol 1986; 3: 101-106.

5. Hedgepeth, C. M., Conrad, L. J., Zhang, J., Huang, H. C., Lee, V. M., \& Klein, P. S. Activation of the Wnt signaling pathway: a molecular mechanism for lithium action. Developmental biology 1997; 185: 82-91.

6. Murray, J. C. Gene/environment causes of cleft lip and/or palate. Clinical genetics 2002; 61: 248-256.

7. Dixon, M. J., Marazita, M. L., Beaty, T. H., \& Murray, J. C. Cleft lip and palate: understanding genetic and environmental influences. Nature Reviews Genetics 2011; 12: 167.

8. The American Society of Health-System Pharmacists "Lithium Salts". Retrieved 2015.

9. Baldessarini, R. J., Tondo, L., Davis, P., Pompili, M., Goodwin, F. K., \& Hennen, J. Decreased risk of suicides and attempts during long-term lithium treatment: a metaanalytic review. Bipolar disorders 2006; 8: 625-639.

10. Brian K. Alldredge; et al. Applied Therapeutics: The Clinical Use of Drugs (10th ed.) 2013; 978-1609.

11. Rhee, J., Kim, R., Kim, Y., Tam, M., Lai, Y., Keum, N., \& Oldenburg, C. E. Maternal caffeine consumption during pregnancy and risk of low birth weight: a dose-response meta- analysis of observational studies. PLoS One 2015; 10: e0132334.

12. Sengupta, P. The laboratory rat: relating its age with human's. International journal of preventive medicine 2013; $4: 624$.

13. Shetty, J. Akhila ,Shyamjith, Deepa and M.C. Alwar Acute toxicity studies and determination of median lethal dose. Current science 2007; 93917-920 :.

14. Tuchmann-Duplessis, H., \& Mercier-Parot, L. Influence du lithium sur la gestation et le développement prénatal du rat et de la souris. CR Soc Biol 1973; 167: 183-186.

15. Loevy, H. T. Lithium ion in cleft palate teratogenesis in CD1 mice. Proceedings of the Society for Experimental Biology and Medicine 1973; 144: 644-646.

16. Meng, L., Wang, X., Torensma, R., Von den Hoff, J. W., \& Bian, Z. Lithium inhibits palatal fusion and osteogenic differentiation in palatal shelves in vitro. Archives of oral biology 2015; 60: 501-507.

17. Lagarto, A., Bellma, A., Tillán, J., Gabilondo, T., Guerra, I., Ocanto,Z.\& González, R. Effect of dolomite oral exposure in Wistar rats during organogenesis period of pregnancy. Experimental and Toxicologic Pathology 2008; 60: 499-504. 
18. Bush, J. O., \& Jiang, R. Palatogenesis: morphogenetic and molecular mechanisms of secondary palate development. Development 2012; 139: 231-243.

19. Giles, J. J., \& Bannigan, J. G. Teratogenic and developmental effects of lithium. Current pharmaceutical design 2006; 12: 1531-1541.

20. Meng, L., Bian, Z., Torensma, R., \& Von den Hoff, J. W. Biological mechanisms in palatogenesis and cleft palate. Journal of dental research 2009; 88: 22-33.

21. Zhou, J., Gao, Y., Lan, Y., Jia, S., \& Jiang, R. Pax9 regulates a molecular network involving Bmp4, Fgf10, Shh signaling and the Osr2 transcription factor to control palate morphogenesis. Development 2013; 140:4709-4718.

22. Einat, H., Kofman, O., Itkin, O., Lewitan, R. J., \& Belmaker, R. H. Augmentation of lithium's behavioral effect by inositol uptake inhibitors. Journal of neural transmission 1998; 105: 31-38.

23. Hopkins, H. S., \& Gelenberg, A. J. Serum lithium levels and the outcome of maintenance therapy of bipolar disorder. Bipolar disorders 2000; 2: 174-179.
24. Gilbert, E. F., \& Pistey, W. R. Effect on the offspring of repeated caffeine administration to pregnant rats. Journal of reproduction and fertility 1973; 34: 495-499.

25. Bullwinkel, J., Baron-Lühr, B., Lüdemann, A., Wohlenberg, C., Gerdes, J., \& Scholzen, T. Ki-67 protein is associated with ribosomal RNA transcription in quiescent and proliferating cells. Journal of cellular physiology 2006; 206: 624-635.

26. Rahmanzadeh, R., Hüttmann, G., Gerdes, J., \& Scholzen, T. Chromophore-assisted light inactivation of pKi-67 leads to inhibition of ribosomal RNA synthesis. Cell proliferation 2007; 40: 422-430.

27. Miyazaki, I., Nagamachi, T., Shinomiya, K., Matsunaga, H., Sendo, T., Kawasaki, H., \& Kitamura, Y. Effects of imipramine and lithium on the suppression of cell proliferation in the dentate gyrus of the hippocampus in adrenocorticotropic hormone-treated rats. Acta Medica Okayama 2010; 46: 219-223.

28. Santarelli, L., Saxe, M., Gross, C., Surget, A., Battaglia, F., Dulawa, S., \& Belzung, C. Requirement of hippocampal neurogenesis for the behavioral effects of antidepressants. Science 2003; 301: 805-809. 